\title{
Resultados iniciales de un programa de cirugía hepato-bilio-pancreática laparoscópica en el Hospital Regional de Talca
}

\author{
César Muñoz C. ${ }^{1,2}$
}

\author{
TRABAJO DE INGRESO
}

\section{Initial results of a laparoscopic hepato-biliary-pancreatic surgery program at the Regional Hospital of Talca}

Introduction: Laparoscopic surgery is the preference access for the treatment of various abdominal pathologies, however, its development in hepato-biliary-pancreatic (HBP) surgery has been slower and heterogeneous. Aim: Present the results of the implementation and development of a laparoscopic HBP surgery program at the Regional Hospital of Talca. Materials and Method: Case series study in which were included all patients submitted to laparoscopic surgery for treatment of HPB pathology as access to the abdominal cavity in the Regional Hospital of Talca between June 1, 2014 and June 30, 2016. Results: There were 42 patients, $25(59.5 \%)$ female. The median age was 58 years (IQ25-75 38-64 years). 22 $(52.4 \%)$ had previous abdominal surgery in most of them by open route. $22(52.4 \%)$ patients were operated on for malignant pathology. The most frequent indication was radical surgery for gallbladder cancer in $10(23.8 \%)$ cases and hepatic hydatidosis $(\mathrm{HH})$ in $7(16.7 \%) .1(2.4 \%)$ patient carrying a $\mathrm{HH}$ required a conversion to laparotomy. $5(11.9 \%)$ presented some postoperative morbidity, 2 of them $>$ Clavien III. The median functional recovery was 1 day (1-2) and the postoperative stay was 3 days (3-4). There was no mortality at 90 days. With a median follow-up of 26.5 (18-33) months, 4 (19\%) of the 21 oncological patients operated on with curative intent presented recurrence of the disease, most of them systemic and 95\% free from recurrence at 24 months. Conclusions: Implementation and development of HBP surgery by laparoscopy is feasible and it can be performed in regional referral hospitals with the same international standards and results.

Key words: laparoscopy; hepatectomy; pancreas; conversion to open surgery.

\section{Resumen}

Introducción: La cirugía laparoscópica es la vía de abordaje de elección para el tratamiento de múltiples patologías abdominales, sin embargo, su desarrollo en la cirugía hepato-bilio-pancreática (HBP) ha sido más lento y heterogéneo. Objetivo: Presentar los resultados de la implementación y desarrollo de un programa de cirugía HBP laparoscópica en el Hospital de Regional de Talca. Materiales y Método: Estudio de serie de casos que incluye a todos los pacientes operados por una patología HBP por vía laparoscópica como acceso a la cavidad abdominal en el Hospital Regional de Talca entre el 1 de junio de 2014 y el 30 de junio de 2016. Resultados: Fueron 42 pacientes, 25 (59,5\%) de sexo femenino. La mediana de edad fue 58 años (IQ25-75 38-64 años). 22 (52,4\%) tuvo una cirugía abdominal previa en la mayoría de ellos por vía abierta. $22(52,4 \%)$ pacientes fueron intervenidos por patología maligna. La indicación más frecuente fue la cirugía radical por cáncer de vesícula biliar en $10(23,8 \%)$ casos y la hidatidosis hepática $(\mathrm{HH})$ en $7(16,7 \%) .1(2,4 \%)$ paciente portador de una HH requirió de una conversión a laparotomía. $5(11,9 \%)$ presentaron alguna morbilidad posoperatoria, 2 de ellos $>$ III de Clavien. La mediana de recuperación funcional fue de 1 día (1-2) y la de estadía posoperatoria de 3 días (3-4). No hubo mortalidad a 90 días. Con una mediana de seguimiento de 26,5 (18-33) meses, 4 (19\%) de los 21 pacientes oncológicos intervenidos con intención curativa presentaron recurrencia de la enfermedad, la mayoría de ellos sistémica y el 95\% está libre de recurrencia a los 24 meses. Conclusiones: La implementación y el desarrollo de la cirugía hepato-bilio-pancreática (HBP) por vía laparoscópica puede efectuarse en hospitales de referencia regional con los mismos estándares y resultados internacionales.

Palabras clave: laparoscopía; hepatectomía; páncreas; conversión a cirugía abierta.
'Hospital Regional de Talca. Talca, Chile.

¿Universidad Católica del Maule. Talca, Chile.

Recibido el 27 de noviembre de 2018 y aceptado para

publicación el 29 de noviembre de 2018.

Correspondencia a: Dr. Cesar Muñoz C. cesarmunozcastro@gmail.com 


\section{Introducción}

La cirugía laparoscópica desde su comienzo en la década de los 80 se convirtió en el abordaje de elección para el tratamiento de varias patologías demostrando disminuir el dolor posoperatorio, disminuir la incapacidad funcional, disminuir la morbilidad y reducir los costos en salud ${ }^{1}$.

La cirugía hepato-bilio-pancreática (HBP) es una disciplina relativamente nueva dentro de la cirugía digestiva, su desarrollo en los últimos años como subespecialidad ha permitido el mejor tratamiento de los pacientes con patología benigna y maligna del hígado, las vías biliares y el páncreas ${ }^{2,3}$. El desarrollo de la cirugía laparoscópica en esta subespecialidad, excluyendo la colecistectomía, ha sido más lento y heterogéneo que en otras disciplinas que pasaron rápidamente de la validación de la seguridad clínica a la evaluación de los resultados perioperatorios y a largo plazo, convirtiéndose, finalmente, en la vía de abordaje de elección para cirugía electiva ${ }^{4}$.

Actualmente, en cirugía HBP se valida la seguridad clínica y los resultados oncológicos para cirugías metástasis hepáticas de cáncer colorrectal ${ }^{5} \mathrm{o}$ la duodeno pancreatoduodenectomía cefálica ${ }^{6,7}$, siendo la evidencia científica escasa respecto al beneficio de esta vía de acceso en estas patologías.

En nuestro país, algunos grupos han desarrollado la cirugía HBP por vía laparoscópica desde el año $2004^{8,9}$ en forma sistemática y principalmente en la región metropolitana, con diversas indicaciones y buenos resultados, siendo incluso en algunas técnicas pioneros en América y el Mundo ${ }^{9-11}$.

El objetivo de este estudio es presentar los resultados de la implementación y desarrollo de un programa de cirugía HBP por vía laparoscópica en el Hospital Regional de Talca.

\section{Material y Métodos}

Estudio de serie de casos en el que se incluye a todos los pacientes intervenidos quirúrgicamente (por el autor) de una patología HBP, en los cuales se utilizó la vía laparoscópica como acceso a la cavidad abdominal en el Hospital Regional de Talca entre el $1^{\circ}$ de junio de 2014 y el 30 de junio de 2016. Se excluyeron todas las colecistectomías laparoscópicas por colelitiasis y las colecistectomías laparoscópicas con colangiografía retrógrada endoscópica simultáneas (Rendez-Vous). Desde junio de 2014, todos los pacientes con patología HBP fueron registrados en una base de datos prospectiva que fue revisada para este estudio.

\section{Técnica quirúrgica}

Se utilizó la posición de Lloyd-Davis en forma rutinaria, con el brazo derecho alineado al cuerpo del paciente para poder realizar colangiografía intraoperatoria en caso necesario. En algunos casos, hemos modificado esta posición a semiprono, para acceder a lesiones del segmento posterior en cirugía hepática.

En la cirugía pancreática se utilizó la misma posición adicionando un cojín toracoabdominal izquierdo para facilitar la disección de la cola de páncreas y el hilio esplénico. La mesa operatoria fue lateralizada en algunos casos para obtener la posición óptima.

En la esplenectomía laparoscópica se utilizó el decúbito lateral derecho con un cojín de Mayo-Robson y anti-Trendelemburg que permite movilizar sin problemas toda la cara posterior y el polo superior del bazo al hacer el abordaje posterior de elección.

Los sistemas de energía utilizados de preferencia fueron, un sistema de corte y coagulación ultrasónico de Covidien ${ }^{\circledR}$ asociado a energía monopolar con pedal a la derecha y bipolar de Microfrance Instruments $^{\circledR}$ con pedal a la izquierda. Se utilizaron 4 a 5 trocares de $5 \mathrm{~mm}$ y $10-12 \mathrm{~mm}$ dependiendo el tipo de intervención a realizar y el neumoperitoneo con aguja de Veress en el punto de Palmer.

De manera estándar se utilizó óptica de $30^{\circ}$ con uno o dos insufladores de $\mathrm{CO} 2$ a una presión de 15 $\mathrm{mmHg}$. Para la extracción de las piezas de mayor tamaño se utilizó una incisión de Pfannestiel con la utilización de un dispositivo Alexis ${ }^{\circledR}$ para protección de la herida.

\section{Variables estudiadas}

Se confeccionó una base de datos específica para este estudio en el cual se consignaron: biodemográficas, índice de masa corporal, morbilidad previa, ASA, antecedentes de cirugía abdominal, tipo de abordaje de cirugía previa, tipo de órgano intervenido, diagnóstico preoperatorio, diagnóstico posoperatorio, requerimientos de Pringle y tiempo, sangrado intraoperatorio, morbilidad operatoria y tratamiento, resultado oncológico de la cirugía, recuperabilidad funcional y estadía posoperatoria.

\section{Morbilidad posoperatoria}

La morbilidad posoperatoria fue consignada como cualquier desviación del curso clínico normal y luego se clasificó según lo propuesto por ClavienDindo $^{12}$ hasta 90 días de posoperatorio.

\section{Seguimiento}

Los pacientes siguieron controles clínicos periódicos y con imágenes cuando la indicación fue 
una neoplasia, algunos de ellos recibieron terapias adyuvantes para el control de su enfermedad. Se consideró cierre de seguimiento el 31 de diciembre de 2017.

\section{Análisis estadístico}

Se confeccionó una base de datos exclusiva para el registro de los datos y el análisis de la serie.

Debido al número de casos y lo heterogéneo de la muestra, las variables continuas se presentan en mediana y rango intercuartil (p25-p75). Las variables categóricas en números absolutos y porcentajes. Para todo el análisis estadístico se utilizó el software STATA 10.0 R.

\section{Aspectos éticos}

Todos los pacientes dieron su consentimiento por escrito para la cirugía luego de una explicación de la técnica quirúrgica a realizar, riesgos y beneficios.

\section{Redacción del manuscrito}

La redacción del manuscrito se ha realizado siguiendo las recomendaciones de la iniciativa STROBE $^{13}$ para el reporte de estudios descriptivos.

\section{Resultados}

En el periodo de estudio se intervinieron 130 pacientes de una patología HPB en el Hospital Regional de Talca. Ochenta y siete de estos procedimientos fueron realizados por el autor, y de ellos 42 cumplieron con los criterios de inclusión. La serie está constituida por $25(59,5 \%)$ pacientes de sexo femenino. La mediana de edad fue de 58 años (38$64)$. Treinta y dos $(76,2 \%)$ pacientes fueron ASA II y $22(52,4 \%)$ tuvieron una cirugía abdominal previa, en la mayoría de ellos por vía abierta. Las características preoperatorias se resumen en la Tabla 1.

La indicación operatoria más frecuente fue el cáncer de vesícula biliar en $10(23,8 \%)$ o la sospecha preoperatoria de cáncer de vesícula (CVB) en 4 $(9,5 \%)$ y la hidatidosis hepática $(\mathrm{HH})$ en $7(16,7 \%)$ pacientes, el detalle de la indicación se muestra en la Tabla 2.

Veintidós $(52,4 \%)$ pacientes se intervinieron por patología maligna, en $21(95,5 \%)$ de ellos se completó la cirugía con intención curativa. En un paciente $(4,5 \%)$ se inició la cirugía con intención curativa por CVB, sin embargo, la biopsia perioperatoria de la linfadenectomía intercavoaórtica resultó positiva y no se continuó con la resección.

La técnica quirúrgica más frecuente fue la cuña hepática con linfadenectomía laparoscópica en 10
$(23,8 \%)$ pacientes seguido por la periquistectomía laparoscópica en 7 (16,7\%) (Figuras 1 y 2). En dos $(4,8 \%)$ pacientes con sospecha preoperatoria de cáncer de vesícula biliar se realizó una colecistectomía laparoscópica con biopsia rápida que informó un carcinoma in situ en uno de los casos y carcinoma $\mathrm{T}_{1 \mathrm{a}}$ en el otro, sin necesidad de completar la cuña

Tabla 1. Características generales de los pacientes

\begin{tabular}{|lc|}
\hline Edad & 58 años $(38-64)$ \\
Sexo n, (\%) & \\
Femenino & $25(59,5)$ \\
Masculino & $17(40,5)$ \\
ASA n, (\%) & \\
I & $9(21,4)$ \\
II & $32(76,2)$ \\
III & $1(2,4)$ \\
Morbilidad preoperatoria n, $(\%)$ & $22(52,4)$ \\
HTA & $13(31)$ \\
Obesidad & $8(19)$ \\
Diabetes mellitus & $5(12)$ \\
Cirrosis & $2(4,8)$ \\
Respiratoria & $1(2,4)$ \\
Renal & $1(2,4)$ \\
Cirugía abdominal previa n, $(\%)$ & $22(52,4)$ \\
Abierto & $14(33,4)$ \\
Laparoscópico & $8(19)$ \\
\hline
\end{tabular}

Tabla 2. "Indicación operatoria"

\begin{tabular}{|c|c|}
\hline Hígado $n,(\%)$ & $17(40,5)$ \\
\hline Hidatidosis hepática & $7(16,7)$ \\
\hline Metástasis hepáticas* & $5(11,9)$ \\
\hline Hepatocarcinoma & $2(4,8)$ \\
\hline Hiperplasia nodular focal & $1(2,4)$ \\
\hline Quiste simple complicado & $1 \quad(2,4)$ \\
\hline Absceso hepático & $1(2,4)$ \\
\hline Vía biliar n, (\%) & $17(40,5)$ \\
\hline CVB & $10(23,8)$ \\
\hline CVB (Sospecha) & $4(9,5)$ \\
\hline Ictericia obstructiva & $2(4,8)$ \\
\hline Quiste de colédoco & $1 \quad(2,4)$ \\
\hline Bazo n, (\%) & $4(9,5)$ \\
\hline PTI & $3(7,1)$ \\
\hline Anemia hemolítica & $1(2,4)$ \\
\hline Páncreas n, (\%) & $3(7,1)$ \\
\hline Pseudoquiste & $2(4,8)$ \\
\hline Tumor de cola & $1 \quad(2,4)$ \\
\hline Duodeno $^{\mp} \mathrm{n},(\%)$ & $1(2,4)$ \\
\hline
\end{tabular}




\section{ARTÍ́CULO ORIGINAL}

Figura 1. Cuña hepática y linfadenectomía laparoscópica. A: Muestreo linfático intercavoaórtico. B: Esquema del muestreo intercavoaórtico C: Visión final de la resección hepática y linfadenectomía. D: AHC: arteria hepática común, AGD: arteria gastroduodenal, AHÍ: arteria hepática izquierda, AHD: arteria hepática derecha, VBP: vía biliar principal, VP: vena porta.

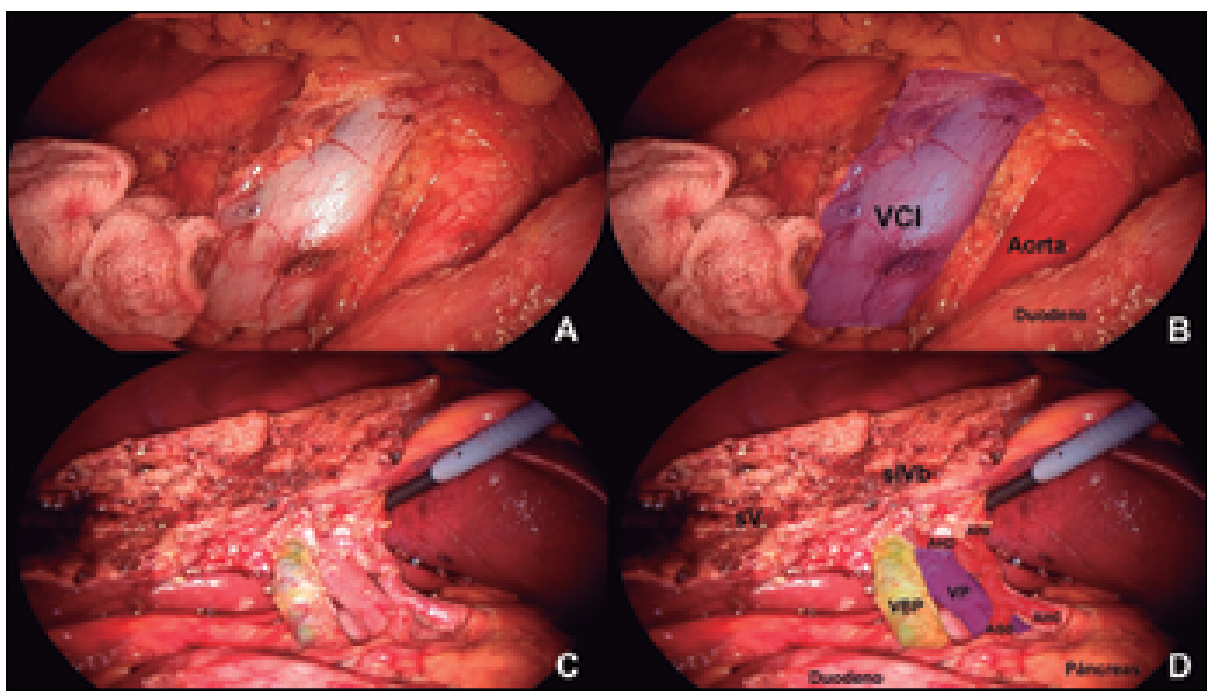

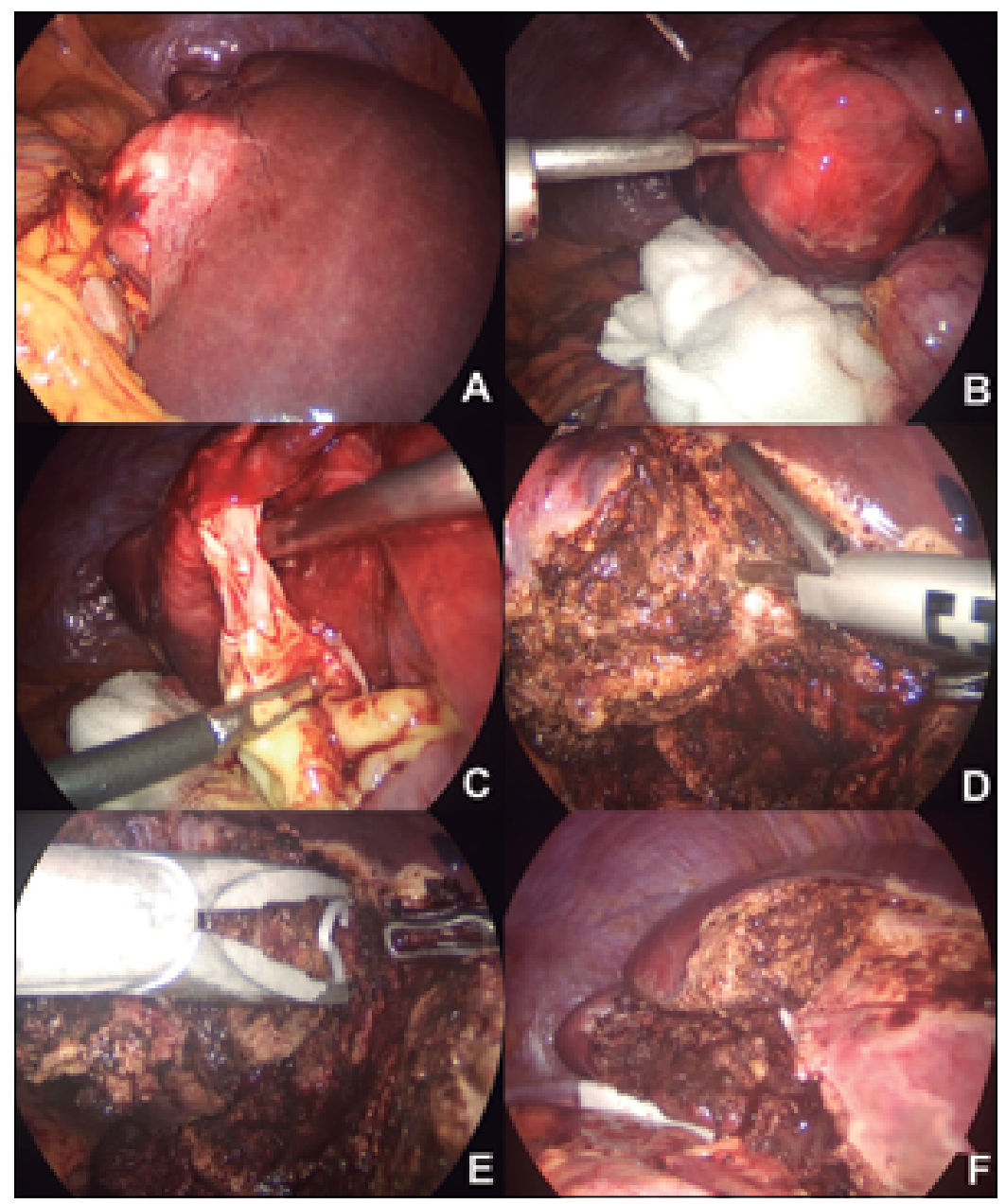

Figura 2. Periquistectomía laparoscópica. A: Quiste hidatídico en segmento VI. B: Punción del quiste, protección del campo con gasas e inyección de solución $\mathrm{NaCl}$ hipertónica. C: Extracción de membrana hidatídica. D: Periquistectomía total con bisturí ultrasónico. E: Control de conductillo biliar con Hem-o-lok. F: Visión final de la periquistectomía total. hepática y linfadenectomía. En cirugía esplénica se realizaron $4(9,5 \%)$ esplenectomías, todas ellas por patología hematológica sin respuesta a tratamiento médico. En cirugía pancreática, 3 intervenciones $(7,1 \%)$ corresponden a una pancreatectomía corporocaudal y $2(4,8 \%)$ cirugías por pseudoquistes pancreáticos. En duodeno, se realizó una cirugía $(2,4 \%)$ de polipectomía laparoscópica con sutura del defecto duodenal tras no poder realizar la polipectomía endoscópica (Tabla 3).

\section{Tabla 3. "Técnicas quirúrgicas"}

\begin{tabular}{|c|c|}
\hline Hígado n, (\%) & $17(40,5)$ \\
\hline Periquistectomía & $7(16,7)$ \\
\hline Hepatectomía anatómica* & $5(11,9)$ \\
\hline Hepatectomía no anatómica & $1(2,4)$ \\
\hline Drenaje y destechamiento & $1(2,4)$ \\
\hline Drenaje de absceso + apendicetomía & $1(2,4)$ \\
\hline Vía biliar n, $(\%)$ & $17(40,5)$ \\
\hline Cuña hepática + linfadenectomía & $11(26,2)$ \\
\hline Coledocoplastía + drenaje de Kehr & $2(4,8)$ \\
\hline Colecistectomía laparoscópica & $2(4,8)$ \\
\hline Resección de vía biliar + HY & $1(2,4)$ \\
\hline Laparoscopía de etapificación & $1(2,4)$ \\
\hline \multicolumn{2}{|l|}{$\begin{array}{l}\text { Bazo n, }(\%) \\
\text { Esplenectomía total }\end{array}$} \\
\hline Páncreas n, (\%) & $4(9,5)$ \\
\hline $\begin{array}{l}\text { Pancreatectomía corporocaudal + } \\
\text { esplenectomía } \\
\text { Cistogastroanastomosis }\end{array}$ & $3 \quad(7,1)$ \\
\hline Cistoyeyunoanastomosis en Y de Roux & $1 \quad(2,4)$ \\
\hline Duodeno $n,(\%)^{\mathrm{F}}$ & $1(2,4)$ \\
\hline
\end{tabular}

*2 segmentectomía lateral, 2 segmentectomía posterior y 1 hepatectomía izquierda. ${ }^{\mp}$ Polipectomia transduodenal. 
Tabla 4. Variables intraoperatorias

\begin{tabular}{|lcccc|}
\hline & Pringle n, (\%) & T Pringle, $\mathbf{~ m i n}^{\S}$ & ${\text { TOP, } \text { min }^{\S}}$ & ${\text { Sangrado, } \mathbf{m l}^{\S}}^{\S}$ \\
Hígado, n: 17 & $3(23,5)$ & $15(15-22,5)$ & $194(124-250)$ & $100(50-100)$ \\
Vía biliar, n: 17 & $8(47)$ & $27(16-37)$ & $357,5(252,5-395)$ & $100(50-100)$ \\
Bazo, n: 4 & - & - & $77,5(55-100)$ & $27,5(10-50)$ \\
Páncreas, n: 3 & - & - & $220(210-230)$ & $50(50-50)$ \\
Duodeno, n: 1 & - & - & 95 & 30 \\
\hline
\end{tabular}

${ }^{\$}$ Mediana, (p25-p75).

Tabla 5. Mortalidad en seguimiento

\begin{tabular}{|cccc|}
\hline & Indicación & Causa de muerte & Seguimiento \\
\hline Caso 1 & CVB & Recurrencia peritoneal & 6 meses \\
Caso 2 & Metástasis hepáticas & Recurrencia pélvica & 12 meses \\
Caso 3 & Metástasis hepáticas & Recurrencia ósea & 21 meses \\
Caso 4 & HCC & Descompensación DHC & 13 meses \\
\hline
\end{tabular}

Un paciente $(2,4 \%)$ portador de una hidatidosis hepática del segmento posterior requirió conversión a una laparotomía de Makuuchi al presentar un tránsito hepatotorácico no pesquisado con los estudios previos y que requirió de una frenorrafia.

Cinco $(11,9 \%)$ presentaron alguna morbilidad posoperatoria, 2 de ellos $>$ III de Clavien y que correspondieron a una filtración biliar después de una periquistectomía que requirió de una colangiografía retrógrada endoscópica y una colección posoperatoria posterior a una cuña hepática que fue tratada con un drenaje percutáneo bajo ecografía. La mediana de recuperación funcional fue de 1 día (1-2) y la de estadía posoperatoria de 3 días (3-4). No se registró mortalidad operatoria en esta serie.

Con una mediana de 26,5 (18-33) meses y un $100 \%$ de seguimiento de la serie, $4(19 \%)$ de los 21 pacientes oncológicos intervenidos con intención curativa presentaron recurrencia de la enfermedad, solo uno de ellos a nivel hepático, el 95\% está libre de recurrencia a los 24 meses. Cuatro (19\%) pacientes han fallecido durante el seguimiento, 3 de ellos por progresión extrahepática (Tabla 5).

\section{Discusión}

La cirugía por vía laparoscópica rompió el paradigma de que una gran laparotomía era garantía de una buena intervención quirúrgica, y que la extensión de la laparotomía era proporcional a una mejor visión del campo operatorio facilitando la capacidad del cirujano para el control de la técnica quirúrgica y las complicaciones intraoperatorias. Cuando la colecistectomía por vía laparoscópica comenzó su desarrollo, experimentados cirujanos escépticos ante esta nueva forma de realizar la colecistectomía, pensaron que el interés sucumbiría ante una excesiva morbilidad de los procedimientos ${ }^{14}$. Contrariamente a lo anterior, en la conferencia del $\mathrm{NIH}$ en el año $1991^{15}$, se concluyó que pese a que la colecistectomía por vía laparoscópica parecía tener una mayor incidencia de lesiones de la vía biliar que la colecistectomía abierta, esta mayor morbilidad podía tolerarse ante los importantes beneficios del nuevo procedimiento menos invasivo. A partir de ese momento, un sin número de instituciones y pacientes quisieron probar la nueva técnica, así como desarrollar nuevos procedimientos a través de pequeñas incisiones ${ }^{16-18}$.

No pasó mucho tiempo hasta que se presentaran series de pacientes intervenidos, comunicando los beneficios del abordaje laparoscópico en reducción de estadía hospitalaria, mejorando la recuperabilidad funcional y disminuyendo el ausentismo laboral; posteriormente, series comparativas validando la utilidad de este abordaje en la cirugía oncológica digestiva.

Un poco al margen de la evolución de la cirugía laparoscópica, la cirugía HPB, pese a tener inicios 
paralelos con otras disciplinas, no se desarrolló de la misma manera. Las razones para esto han sido: la necesidad de control vascular durante una resección hepática mayor, la necesidad de habilidades laparoscópicas avanzadas para el control de complicaciones como sangrados o fugas biliares, la dificultad técnica de una linfadenectomía del pedículo hepático o del tronco celíaco, o las complejas anastomosis que necesitan ser reconstruidas durante una duodenopancreatectomía cefálica, por dar un ejemplo ${ }^{19}$.

La visión y entusiasmo de cirujanos pioneros como Brice Gayet ${ }^{20}$ y centros $^{6}$ que tuvieron una visión más en perspectiva e integral respecto al desarrollo de las técnicas mínimamente invasivas, son un ejemplo como el trabajo metódico y sostenido permite las mismas curvas de aprendizaje en el desarrollo de la cirugía HPB mínimamente invasiva ${ }^{21}$ y cuyos resultados no son sólo comparables, sino incluso superiores en algunos aspectos respecto a la cirugía abierta.

La implementación de programas de entrenamientos específicos permite reducir las curvas de aprendizaje para habilidades avanzadas como, por ejemplo, sutura laparoscópica o el control vascular durante una resección hepática por laparoscopia ${ }^{22,23}$. Actualmente, centros pioneros en cirugía por vía laparoscópica abdominal, han logrado llegar a un $90 \%$ de las resecciones hepáticas mediante abordaje laparoscópico con excelentes resultados ${ }^{24}$.

En patologías tan frecuentes como la colecistocoledocolitiasis aún se discute si el abordaje de la vía biliar totalmente laparoscópico es la mejor alternativa de tratamiento para la resolución de la colecistocoledocolitiasis por su complejidad técnica y no por su efectividad, que ha demostrado ser comparable a la cirugía abierta manteniendo la integridad del esfinter de $\mathrm{Oddi}^{25}$. Respecto a lo anterior, podemos decir que la evidencia disponible no permite demostrar que el tratamiento totalmente laparoscópico sea mejor que el combinando abordaje laparoscópico y endoscópico, solo que en la selección del tratamiento se debe considerar el entrenamiento quirúrgico, la disponibilidad de endoscopia y el riesgo quirúrgico del paciente ${ }^{26}$.

En una línea paralela, la cirugía pancreática ha intentado realizar su evolución confirmando que probablemente los excelentes resultados reportados por Kendrick M. et al. ${ }^{27}$, no son reproducibles para todos los centros, y vienen a confirmar que se deben reunir algunas características para que estos procedimientos puedan ser realizados con garantías oncológicas y de seguridad clínica para los pacientes.

En términos oncológicos, muchas veces se cuestiona si el abordaje mínimamente invasivo es suficientemente bueno en la cosecha ganglionar en comparación con la cirugía clásica abierta ${ }^{28}$. En esta serie, la cirugía más frecuentemente realizada fue la cuña hepática y linfadectomía por cáncer de la vesícula biliar donde desde el punto de vista oncológico se ha cumplido con todos los principios que se han sugerido como necesarios para una buena cirugía oncológica ${ }^{29,30}$. El seguimiento que hemos logrado hasta el momento, junto con los resultados, sugieren que no vamos por un mal camino ${ }^{31}$. Un beneficio que es interesante de considerar y que en nuestra serie solo corresponde a un paciente, es el evitar una laparotomía para realizar una cirugía que, finalmente, terminará solo como una etapificación.

Un punto importante al momento de iniciar un programa de cirugía por vía laparoscópica en HPB y que se experimentó durante la implementación y el desarrollo de esta serie clínica es la necesidad de instrumental, dispositivos técnicos, sutura mecánica que encarecen el procedimiento quirúrgico. Esta situación muchas veces es difícil de explicar a directivos y administrativos que ven poco beneficio en la reducción del costo en días cama o la necesidad de cuidados intensivos perioperatorios, menos beneficio directo en mejorar la recuperabilidad funcional precoz y disminuir el ausentismo laboral.

Una de las limitantes técnicas que se tuvo en el desarrollo de esta serie clínica fue la ausencia de una transductor ecográfico laparoscópico para poder realizar una ecografía intraoperatoria que es fundamental para poder realizar resecciones hepáticas mayores o anatómicas segmentarias en pacientes con metástasis hepáticas de cáncer colorrectal y así favorecer el concepto de cirugía preservadora de parénquima en este grupo de pacientes. La adquisición de este dispositivo seguramente nos permitirá aumentar la complejidad y el número de cirugías de este tipo realizadas en estos pacientes. Si bien existen técnicas alternativas para reemplazar o utilizar un transductor regular en cirugía por vía laparoscópica, tanto la visión como la posibilidad de realizar una incisión baja tipo Pfannestiel para la extracción de grandes piezas, permiten que este instrumento sea el ideal durante la ejecución de esta cirugía.

Por último, y como una manera justificar estas técnicas, un análisis de costo beneficio evidencia que un mayor número de cirugías laparoscópicas realizadas podría reducir un 50\% la ocupación de días cama equiparando los mayores costos del procedimiento quirúrgico en cirugías de reflujo gastroesofágico y colorrectal ${ }^{32,33}$. Recientemente, un estudio prospectivo aleatorizado comparando costos en el tratamiento de metástasis hepáticas de cáncer colorrectal, demostró un beneficio en disminución 
de costos generales y ajustados por calidad de vida de cada paciente intervenido mediante abordaje laparoscópico, pese a que, inicialmente, los costos de la cirugía por vía laparoscópica fueron un poco mayores $^{34}$.

\section{Agradecimientos}

A mis profesores que han invertido tiempo en enseñarme lo necesario para poder progresar en esta carrera quirúrgica, mi gratitud a ellos en: Universidad Católica de la Santísima Concepción, Universidad de La Frontera, Pontificia Universidad Católica de Chile y Hospital Clínic de Barcelona. A los pacientes por la confianza entregada y la oportunidad de tratarlos de sus padecimientos. Al Hospital Regional de Talca (anestesiólogos, enfermeras, paramédicos y administrativos) y los cirujanos del equipo de Cirugía Digestiva; sin ellos y su apoyo constante este trabajo clínico indudablemente no habría sido posible. Finalmente, a mi familia que sin su compañía, cariño y comprensión, todo habría sido probablemente más solitario y difícil de llevar, son una diaria inspiración.

\section{Responsabilidades éticas}

Protección de personas y animales. Los autores declaran que para esta investigación no se han realizado experimentos en seres humanos ni en animales.

Confidencialidad de los datos. Los autores declaran que en este artículo no aparecen datos de pacientes.

Conflictos de interés: no hay.

\section{Bibliografía}

1. Miyasaka Y, Nakamura M, Wakabayashi G. Pioneers in laparoscopic hepatobiliary-pancreatic surgery. J Hepatobiliary Pancreat Sci. 2018;25:109-11.

2. Abdalla E. Advances in hepato-pancreato biliary surgery. Expert Rev Gastroenterol Hepatol. 2011;5:457-60.

3. He VJ. Professor Henri Bismuth: the past , present and future of hepatobiliary surgery. Hepatobiliary Surg Nutr. 2013;2:236-8.

4. Tan-Tam C, Chung S. Minireview on laparoscopic hepatobiliary and pancreatic surgery. World J Gastrointest Endosc. 2014;6:60-7.

5. Beppu T, Yamamoto M. Laparoscopic Versus Open Liver Resection for Colorectal Liver Metastases-Which Is a More Suitable Standard Practice? Ann Surg. 2018;267:208-9.

6. Palanivelu C, Senthilnathan P, Sabnis S, Babu N, Srivatsan, Gurumurthy Anand VN, et al. Randomized clinical trial of laparoscopic versus open pancreatoduodenectomy for periampullary tumours. Br J Surg. 2017;104:1443-50.

7. Palanivelu C, Kyoichi T, Mohammad AH, David AK, Go W, Agarwal A, et al. International Summit on Laparoscopic Pancreatic Resection (ISLPR) "Coimbatore Summit Statements". Surg Oncol. 2018;27:A10-5.
8. Sanhueza M, Reyes J, Díaz A. Hepatectomía derecha laparoscópica. En: LXXVIII Congreso Chileno e Internacional de Cirugía. 2005. p. 161-2.

9. Jarufe NC, Fernández JF, Boza CW, Navarrete FC, Escalona AP, Funke RH, et al. Pancreatoduodenectomía totalmente laparoscópica: Técnica quirúrgica y experiencia inicial. Rev Chil Cir. 2009;61:33-8.

10. Gumbs AA, Jarufe N, Gayet B. Minimally invasive approaches to extrapancreatic cholangiocarcinoma. Vol. 27, Surgical Endoscopy and Other Interventional Techniques. 2013. p. 406-14.

11. De Aretxabala X, Leon J, Hepp J, Maluenda F, Roa I. Gallbladder cancer: Role of laparoscopy in the management of potentially resectable tumors. Surg Endosc. 2010;24:2192-6.

12. Dindo D, Demartines N, Clavien P-A. Classification of Surgical Complications. Ann Surg. 2004;240:205-13.

13. Vandenbroucke JP, von Elm E, Altman DG, Gotzsche PC, Mulrow CD, Pocock SJ, et al. Strengthening the Reporting of Observational Studies in Epidemiology (STROBE): Explanation and elaboration. PLoS Med. 2007;4:e297.

14. Targarona EM. N.O.T.E.S: ¿qué hemos aprendido? Cir Esp. 2012;90:1-3

15. National Institutes of Health Consensus Development Conference Statement on Gallstones and Laparoscopic
Cholecystectomy. Am J Surg. 1993;165:390-8

16. Gagner M, Rheault M, Dubuc J. Laparoscopic partial hepatectomy for liver tumor. Surg Endosc. 1992;6:99.

17. Potvin M, Gagner M, Pomp A. Laparoscopic Roux-en-Y gastric bypass for morbid obesity: a feasibility study in pigs. Surg Laparosc Endosc. 1997;7:2947.

18. Gagner M, Pomp A, Todd Heniford B, Pharand D, Lacroix A. Laparoscopic adrenalectomy: Lessons learned from 100 consecutive procedures. Ann Surg. 1997;226:238-47.

19. Takaori K, Tanigawa N. Laparoscopic pancreatic resection: The past, present, and future. Surg Today. 2007;37:535-45.

20. Gumbs A, Gayet B. The medical and surgical department of digestive diseases and minimally invasive HPB fellowship at IMM. Hpb. 2007;9:470-1.

21. Vibert E, Perniceni T, Levard H, Denet C, Shahri NK, Gayet B. Laparoscopic liver resection. Br J Surg. 2006;93:67-72.

22. Castillo R, Buckel E, León F, Varas J, Alvarado J, Achurra P, et al. Effectiveness of learning advanced laparoscopic skills in a brief intensive laparoscopy training program. J Surg Educ. 2015;72:648-53.

23. Varas J, Mejía R, Riquelme A, Maluenda F, Buckel E, Salinas J, et al. Significant transfer of surgical skills obtained with an advanced laparoscopic training program 
to a laparoscopic jejunojejunostomy in a live porcine model: Feasibility of learning advanced laparoscopy in a general surgery residency. Surg Endosc Other Interv Tech. 2012;26:3486-94.

24. Gumbs A, Gayet B. Adopting Gayet's Techniques of Totally Laparoscopic Liver Surgery in the United States. Liver Cancer 2013;2:5-15.

25. Zhu JG, Han W, Guo W, Su W, Bai ZG, Zhang ZT. Learning curve and outcome of laparoscopic transcystic common bile duct exploration for choledocholithiasis. Br J Surg. 2015;102:1691-7.

26. Pekolj J. Tratamiento de la litiasis coledociana por vía laparoscópica. Continúa la controversia. Cirugía Española 2012;90:144-6.

27. Kendrick M, Cusati D. Total laparoscopic pancreaticoduodenectomy: feasibility and outcome in an early experience. Arch
Surg. 2010;145:19-23.

28. Ong C, Leung K, Nussbaum D, Sun Z, Gloor B, Blazer D, et al. Open versus laparoscopic portal lymphadenectomy in gallbladder cancer: is there a difference in lymph node yield? HPB (Oxford). 2018;20:505-13.

29. Muñoz C, Marino C, Morales E. Totally laparoscopic radical cholecystectomy (lymphadenectomy and segment $\mathrm{IVb}-\mathrm{V}$ liver resection) after incidental gallbladder carcinoma (with video). J Visc Surg. 2018;155:243-4.

30. Aloia TA, Járufe N, Javle M, Maithel SK, Roa JC, Adsay V, et al. Gallbladder Cancer: Expert consensus statement. HPB (Oxford). 2015; 17:681-90.

31. Castro CM, Santibáñez SP, Rivas TC, Cassis NJ. Totally Laparoscopic Radical Resection of Gallbladder Cancer: Technical Aspects and Long-Term
Results. World J Surg. 2018;42:2592-8.

32. Jordan J, Dowson H, Gage H, Jackson $\mathrm{D}$, Rockall T. Laparoscopic versus open colorectal resection for cancer and polyps: A cost-effectiveness study. Clin Outcomes Res. 2014;6:415-22.

33. Grant AM, Boachie C, Cotton SC, Faria R, Bojke L, Epstein DM, et al. Clinical and economic evaluation of laparoscopic surgery compared with medical management for gastro-oesophageal reflux disease: 5-year follow-up of multicentre randomised trial (the REFLUX trial). Health Technol Assess (Rockv). 2013;17:1-167.

34. Fretland ÅA, Dagenborg VJ, Bjørnelv GMW, Kazaryan AM, Kristiansen R, Fagerland MW, et al. Laparoscopic Versus Open Resection for Colorectal Liver Metastases. Ann Surg. 2018;267:199-207.

\section{Comentario de trabajo ingreso}

Agradezco a la Sociedad de Cirujanos la oportunidad de realizar el comentario del trabajo de ingreso del Dr. César Muñoz Castro, quien presenta "Resultados iniciales de un programa de Cirugía Hepato-Bilio-Pancreática Laparoscópica en el Hospital Regional de Talca" de la Unidad de Cirugía Digestiva de dicho Centro y del Departamento de Cirugía de la Universidad Católica del Maule.

Se plantea como objetivo de su trabajo presentar los resultados de la implementación y desarrollo inicial de un programa por vía laparoscópica en su centro. Cabe señalar que refiere en la introducción de su presentación las ventajas evidentes de esta aproximación en múltiples cirugías abdominales y en varias de la esfera HPB. Para esto utiliza una serie de casos clínicos en forma descriptiva con una cantidad de pacientes adecuadas para la región (42 pacientes) en 2 años. Se preocupó de instaurar en su sistema público los elementos necesarios y adecuados para realizar cirugía laparoscópica HPB en buen nivel como coagulador bipolar, coagulador ultrasónico, óptica de 30 grados y en la actualidad solicitando un equipo de alta calidad de ultrasonido laparoscópica.

Se consignaron las complicaciones con la clasificación Clavien-Dindo en forma muy adecuada, fijándose un límite en la mortalidad posoperatoria dentro de 90 días como es lo recomendado para evaluar los resultados de las intervenciones.

Lo que echo de menos es el porcentaje de seguimiento a los 42 meses, que debió ser cercano al 80 a $90 \%$ según los datos (no explicitado eso sí en el manuscrito).

Según lo notificado, obtuvo por escrito el consentimiento informado para el estudio y la técnica quirúrgica utilizada, cosa que es absolutamente necesario en la actualidad para la mantención de los derechos del paciente.

Dentro de los pacientes incluidos me parece que si bien la esplenectomía laparoscópica (4 en esta serie de casos) es hermosa cirugía, dentro de las cirugías laparoscópicas es de baja complejidad, creo que no pertenece a una cirugía HPB propiamente tal, pero si una cirugía laparoscópica cuyo goldstandard para este órgano es esta vía de acceso al abdomen, pero podría ser incluida en algún trabajo que no incluya en el título la exclusividad de HPB. Debo dejar explícito que por aclaración del autor, las incluyó en su comunicación por el hecho que este tipo de intervención se había dejado de realizar en dicho centro el año 2011, por lo que me parece perfecto le reiniciación de dicha intervención.

En la actualidad, sin duda que el gold-standard en algunas cirugías HPB como son la pancreatec- 
tomía corporocaudal y/o distal, segmentectomía hepática lateral izquierda (segmentos 2 y 3 ) y los segmentos laterales y periféricos derechos es la aproximación laparoscópica; incluso me atrevería a decir que en las pocas lesiones hepáticas benignas sólidas posiblemente las lobectomías derecha o izquierda llegarán a ser todas por vía laparoscópica excepto las de mayor complejidad.

Aún está por demostrarse y en deuda esta aproximación laparoscópica para la duodenopancreatectomía cefálica y otras cirugías como la del tumor de Klatskin.

Muy importante es y fue la formación y preparación de Dr. César Muñoz, quien realizó sus residencias en centros formativos con entrenamiento tanto abierto como laparoscópico nacionales e internacionales como lo es el Hospital Clinic de Barcelona por dos años entre 2016 y 2018, específicamente en Cirugía HPB y trasplante de hígado y riñón; por lo que es muy reconfortante que lo implemente y mantenga en el caso de trasplante renal en una ciudad distinta a la capital del país, donde sin duda adolecía de este grado de complejidad en cirugía HPB.
La morbilidad en cantidad y en tipo e complicaciones es la adecuada para cualquier centro nacional o internacional en cirugía laparoscópica.

En relación al seguimiento de los 21 pacientes oncológicos intervenidos con intención curativa tiene una mediana de 26,5 meses (18-33 meses) lo que me imagino completará en su totalidad un mínimo de 5 a 10 años, pero la mediana que presenta es más del período de recurrencia por todos temidos en tumores malignos digestivos (primeros 24 meses).

Con todo lo comentado y con el trabajo realizado por el Dr. César Muñoz Castro tengo el honor y el agrado de recomendar a este cirujano digestivo como miembro titular de la Sociedad de Cirugía de Chile y no me queda más que felicitarlo e incentivarlo a continuar el desarrollo en su región y en el país al alero de la Sociedad de Cirujanos y del Departamento de Hígado, Páncreas y Vías Biliares (HPB) de dicha Sociedad.

Felicitaciones por su labor realizada y por su trabajo presentado.

Dr. Jaime Castillo Koch 